\title{
SOCIEDADES EN COLAPSO: LA TRANSICIÓN DEL CLÁSICO AL EPICLÁSICO EN TEOTIHUACAN
}

\author{
SOCIETIES COLLAPSE: THE TRANSITION FROM CLASSICAL TO \\ EPICLASSIC TIMES IN TEOTIHUACAN
}

\author{
Natàlia Moragas Segura*
}

\begin{abstract}
El colapso de Teotihuacan es uno de los más importantes eventos en la arqueología mesoamericana, ya que marca un cambio cultural y sociopolítico en un extenso territorio. Es también un tema muy popular entre los investigadores que han hipotetizado una serie de diferentes razones para explicar por qué colapsó. Más que buscar las razones, más allá de las causas, en este trabajo interesa más considerar los procesos sociopolíticos detectados por medio de las excavaciones de los últimos años. Teotihuacan.

Palabras claves: arqueología de Teotihuacan, colapso de sociedades complejas, transición del clásico al epiclásico,
\end{abstract}

The collapse of Teotihuacan is one of the most important events in Mesoamerican archaeology as it marks a cultural and sociopolitical change in this extensive territory. It is therefore a very popular subject among researchers, who have put forward a number of different reasons to explain why it collapsed. Rather than looking for the reasons behind the great crisis that befall this culture, this paper focuses on the sociopolitical processes detected through excavations carried out over recent few years.

Key words: archaeology, collapse of complex societies, classic-epiclassic transition.

\section{Introducción}

Para los investigadores de las ciencias sociales y humanas el estudio del colapso de las sociedades complejas es uno de los tópicos de investigación que se ha estudiado de manera recurrente desde principios del siglo XX hasta la actualidad. Aún supone hoy un reto comprender cómo una sociedad, aparentemente estable en sus formas (culturales, políticas, económicas y sociales), decae de manera irremediable. Este es un tema de debate e investigación actual que trasciende el campo de lo meramente académico. Sin duda alguna, el colapso es un tópico querido también por el público en general y que es utilizado por los medios de comunicación para perpetuar ese imaginario del pasado romántico de civilizaciones desaparecidas o admoniciones de futuros catastrofistas si no aprendemos las lecciones del pasado.

Este artículo trata del colapso de la cultura teotihuacana y de su reconfiguración en un nuevo orden mesoamericano. Considerando que Teotihuacan, durante el período Clásico, marcó el desarrollo cultural de gran parte del altiplano mexicano y que abarcó un amplio radio de influencia en otras culturas mesoamericanas contemporáneas, su colapso supone una cuestión fundamental para el desarrollo posterior de esta área cultural. No obstante, el estudio del colapso de la cultura teotihuacana si bien es un tópico conocido y aceptado por los mesoamericanistas, siento que se ha tratado en menor medida.

El final de Teotihuacan es uno de los ejemplos clásicos de la historiografía arqueológica sobre el colapso de las sociedades complejas. El hecho de que una sociedad aparentemente activa, vibrante, en pleno esplendor de su desarrollo cultural colapse de manera inmediata ha llenado páginas de bibliografía especializada y de difusión. Las diversas hipótesis que se han manejado han oscilado entre las causas monocausales a multicausales, y entre ellas una combinación de factores internos y externos (Moragas, 2009). De propuestas invasionistas y difusionistas a conflictos sociales internos entre el pueblo y las élites. En los últimos años se han incorporado modelos que enfatizan relaciones de convivencia entre los coyotlatelcos y los teotihuacanos y los conflictos entre élites como causa de la crisis social de la ciudad (Moragas, 2005). Actualmente el consenso general de los investigadores es que el colapso es un fenómeno político-ideológico que surge en el seno de las élites teotihuacanas y que afectará en mayor o menor medida en el conjunto de la población.

* Universitat de Barcelona, Departamento de Antropología Social, Historia de América y África, España. Correo electrónico: nataliamoragas@ub.edu 
Sin embargo, debemos poder ir rediseñando este modelo incorporando más factores para su análisis y evaluación. En este trabajo se va a considerar el concepto de crisis en el mundo teotihuacano y las evidencias de cambios políticos dentro de la ciudad. Mi propuesta considera que debemos tener en cuenta dos actores que responderán de manera distinta a la crisis final de la cultura teotihuacana debido a sus diferentes momentos históricos propios. Por un lado, los teotihuacanos que después de 600 años dominando ideológicamente el altiplano mexicano no parecen ser capaces de reconducir la situación. Por otra parte los coyotlatelcos, los últimos pueblos que llegan al valle de México y que se van incorporando a la órbita teotihuacana a partir del 450-500 d.C.

\section{Teotihuacan: un mundo ordenado}

Teotihuacan se encuentra situado en el noroeste de la cuenca de México en un pequeño valle situado a $19^{\circ} 34^{\prime} \mathrm{N}, 99^{\circ} 40 \mathrm{~W}$ con elevaciones que oscilan entre los 2.400 y $3.100 \mathrm{msnm}$. El régimen de lluvias va de finales de mayo hasta finales de octubre y durante el año se suceden unas temperaturas medias de $5-15{ }^{\circ} \mathrm{C}$ en cotas superiores y un promedio de
$12-18{ }^{\circ} \mathrm{C}$ en cotas inferiores a $2.800 \mathrm{msnm}$. El área que ocupa la ciudad se encuentra circunscrita por el cerro Gordo al norte y por la sierra Patlachique al sur (Mooser, 1968; McClung de Tapia y otros, 1996:21).

Sin duda alguna Teotihuacan reproduce un modelo cósmico del mundo del altiplano mesoamericano a fines del preclásico superior y principios del clásico. Es posible que nos sea muy complicado saber la causa exacta del origen de la ciudad, pero las sucesivas investigaciones llevadas a cabo en Teotihuacan, Cuicuilco y más recientemente en el valle poblano-tlaxcalteca nos muestra que la movilidad de las poblaciones del altiplano fueron determinantes para la constitución de la urbe (Carballo,2009; Carballo y Pluckhahn, 2007). Hay diversos factores involucrados como serán los movimientos volcánicos y tectónicos de fines del preclásico, el desarrollo cultural de dichas poblaciones, el cambio de las redes de intercambio y la propia disposición de la futura ciudad, cuyo valle posiblemente sea reinventado bajo una particular cosmovisión. Podría ser algo arriesgado afirmar que el surgimiento de Teotihuacan es consecuencia exclusiva de factores externos al propio valle, ya que

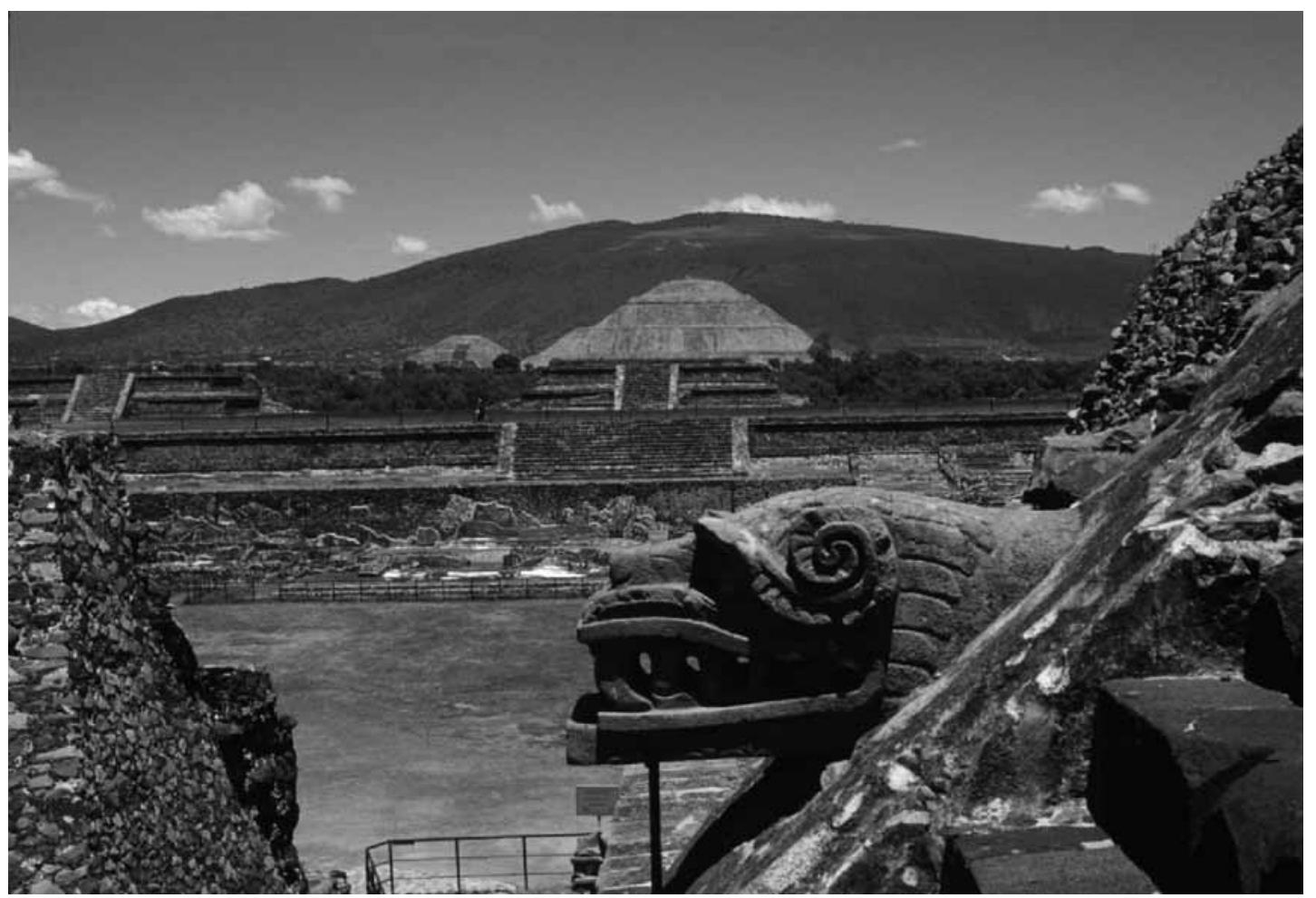

Figura 1. Pirámides del Sol y de la Luna desde el Templo de la Serpiente Emplumada (Foto Miguel Morales INAH-ZAT). 
tenemos pocas evidencias de poblaciones preteotihuacanas. Y decimos arriesgado porque los datos arqueológicos nos son esquivos debido probablemente al impacto que supone la construcción de la urbe y las sucesivas remodelaciones arquitectónicas. El asentamiento de más de 100.000 personas durante varios siglos supone un impacto continuado en el territorio más cercano y, por lo tanto, complica el hallazgo de fases preteotihuacanas. En todo caso sí que podemos considerar que el origen de Teotihuacan es la resolución de una gran crisis de parte de la población del centro de México.

Varios factores clave contribuyen a la rapidez de la consolidación del modelo teotihuacano. Tradicionalmente se ha manejado el control de la sierra de las Navajas y el monopolio de la explotación de la obsidiana verde como el motor económico de la ciudad (Spence,1981,1984,1987). A ello hay que añadirle el carácter sagrado de la ciudad con la cueva de la pirámide del Sol como parte de un axis mundi (Cowgill, 1988). René Millon conceptualizó el modelo de peregrino-templo-mercado en torno a los conjuntos de tres templos como parte de un genuino patrón teotihuacano (Millon, 1973,1976). Hoy sabemos que dicho modelo debe de ser matizado, ya que se conformó en un momento en el que se consideraba a Teotihuacan como una sociedad eminentemente teocrática y se aplicaban modelos reconocidos en otras culturas como la mexica o el Próximo Oriente. Hoy proponemos un sistema de linajes en el que la organización teotihuacana comprendió un modelo de asentamiento diferenciado de otras sociedades contemporáneas ${ }^{1}$ (Manzanilla, 2006). Los teotihuacanos eran conscientes que ocupaban un espacio privilegiado desde el punto de vista cosmogónico y lo reprodujeron durante seiscientos años en lo que podríamos definir actualmente como un excelente diseño de marketing corporativo.

Creo que es interesante para replantearse algunas de las cuestiones claves de Teotihuacan considerar cómo se ha escrito la historia de la misma y la imagen que se ha construido y que se ha difundido. Uno de los argumentos que creo que han marcado la visión de esta ciudad es la dificultad de poder establecer una historia política de la misma (Cowgill, 2008). En las representaciones humanas se identifica más la clase y la función que realizan que la individualidad de los mismos (Cowgill, 1997, 2008; Manzanilla, 2005;2006), por lo que no podemos por ahora identificar con claridad nombres de gobernantes en la misma medida que se han podido hacer en la cultura maya por ejemplo. Las causas son conocidas desde antiguo por los investigadores y se refieren a la aparente falta de individualidad en las representaciones teotihuacanas o como en la falta de una escritura comparable a las de otras culturas mesoamericanas. La escritura en las sociedades antiguas tiene varias funciones, pero se ha vinculado a la gestión de los bienes de la ciudad y a la representación del poder de las élites. En el caso mesoamericano, la escritura parece vinculada a las representaciones políticas y el ejercicio del mismo por parte de las élites y por ello aparece a menudo vinculado a la arquitectura monumental y en contextos de élites y poder.

Es una problemática clave, ya que ambos factores, combinados en una misma cultura, nos complica sobremanera la comprensión de las acciones políticas de las élites y sus decisiones, que son elementos necesarios para conocer la historia de la ciudad. Si esta cuestión es importante para cualquier cultura, aún lo es más cuando además se considera que Teotihuacan marca el devenir político y cultural de gran parte de Mesoamérica durante los primeros quinientos años de la era cristiana.

\section{Crisis y colapso: una evaluación historiográfica}

Historiográficamente durante gran parte del siglo XX el período Clásico del altiplano se construyó a base de lo que se conocía de la cultura teotihuacana, ya que se había vinculado el desarrollo histórico cultural del altiplano al poder ejercido por los teotihuacanos en el territorio. Dicho conocimiento venía determinado por el propio desarrollo de la arqueología en su momento histórico.

Resulta interesante observar que desde un principio los investigadores e historiadores de la antigüedad teotihuacana consideraron que la ciudad fue conquistada por los toltecas en 674 (Chavero, 1984) y/o destruida por tribus invasores o abandonadas (Batres, 1889). Y acorde a los tiempos, las propuestas difusionistas-invasionistas son las que tuvieron una mayor popularización debido a las evidencias arqueológicas más sobresalientes, y evidentes, como son: el incendio del centro ceremonial, el saqueo de las estructuras y el cambio de la cultura material. Sin querer incidir en demasía, hemos considerado que en un primer momento podríamos dividir las interpretaciones sobre el 
colapso de Teotihuacan en dos grandes factores, como son los externos y los internos.

Los factores externos combinan básicamente casuísticas difusionistas y/o invasionistas, así como la idea de que la sociedad teotihuacana no puede sobrevivir a factores derivados de cuestiones ajenas a su estructura cultural. Sin duda alguna, la presencia de un complejo cerámico completamente ajeno al existente supuso la presencia de un grupo cultural diferente que se impone bajo la fuerza y cuya consecuencia serán los incendios y saqueos que se detectan. Los coyotlatelcos originarios probablemente en el occidente/bajío de México (Acosta, 1972; Braniff, 1972) o tal vez otomíes (Jiménez Moreno, 1966: 51-53) atacarán y destruirán a la ciudad que será abandonada por los propios teotihuacanos diluyéndose en el continuo cultural mesoamericano. Sin embargo, progresivamente se fueron declarando algunas dudas tanto por las magnitudes de las fuerzas coyotlatelcas armadas que debieron de atacar y conquistar una ciudad con una estructura urbanística muy compleja que hubiera requerido de una estrategia de conquista de calle a calle y de plaza a plaza. Por otro lado, no queda claro de qué manera los teotihuacanos desaparecen de la ciudad porque los coyotlatelcos no toman los elementos del poder teotihuacano como un ejemplo de legitimación local. Otras propuestas, de carácter externo, tuvieron mucha aceptación, como son las causas medioambientales y climáticas que van desde planteamientos catastrofistas hasta fenómenos climáticos de carácter continental (Lorenzo, 1968; Messenger, 1990; Millon, 1973; Palerm y Wolf, 1972; Siebe et al., 1996: 402). La idea de un cambio climático global resultaba atractiva, pero complicada a la hora de poder establecer datos e indicadores arqueológicos claros; entre otras cosas, por la propia morfología del valle de Teotihuacan que hace difícil la conservación de macrorrestos vegetales y polínicos que pudieran afectar a la ciudad. Otro grupo de hipótesis inciden en el desgaste que supuso el sostenimiento de una macrociudad como Teotihuacan en el propio valle y en la propia cuenca de México (Cook, 1947; Lorenzo, 1968: 57; Mooser, 1968: 37). En el fondo, este corpus de teorías están bajo la influencia que tuvo el trabajo del Teotihuacan Valley Project dirigido por William Sanders y que propugnaba que el rápido crecimiento de Teotihuacan sobreexplotó el territorio circundante e hizo la ciudad dependiente de una amplia periferia (Sanders, 1979). A pesar de todo esto, los factores externos por ellos solos no terminan de explicar el papel de los coyotlatelcos ni tampoco de la caída de la ciudad y el abandono de la misma, debido a que no hay evidencias concluyentes de un cambio climático tan extremo. Es por ello que otro grupo de investigadores sugirieron que los factores que explican el colapso de Teotihuacan deben encontrarse interno a la ciudad y sobre todo en el papel de las élites, cuya función principal dentro de la sociedad sería la de garantizar la estabilidad del ciclo agrícola gracias a su especial vinculación con los dioses y el control y dominio de los rituales necesarios para el sostenimiento de la ciudad. Asimismo se añaden nuevos elementos como son el progresivo militarismo en las representaciones teotihuacanas, la inestabilidad regional y el contraste campo-ciudad (Matos, 1996; Millon, 1967, 1973; Sanders, 1964; Sanders y Price 1968; Webb, 1978). Finalmente, la pérdida de las rutas comerciales, el desprestigio de la urbe y las emergentes élites regionales suponen un proceso de desintegración del poder teotihuacano y el sistema ideológico-político que la sustentaba (Cohodas, 1989; Litvak, 1970, 1978; Sanders, 1956, 1964; Sanders y Price, 1968).

Los años 90 fueron un revulsivo para el estudio de las fases posteriores al colapso de Teotihuacan gracias a las investigaciones derivadas del proyecto sobre el inframundo teotihuacano dirigido por Linda Manzanilla (UNAM) y las del proyecto Especial Teotihuacan 92-94 (Manzanilla y Arrellín, 1999; Moragas, 1995). Ambos proyectos se iniciaron con finalidades distintas, el primero con clara vocación de investigación acerca del sistema de túneles y cuevas, mientras que el segundo fue consecuencia de un salvamento para condicionar el acceso a la puerta 5. En el primer proyecto el interés principal consistía en encontrar fases tempranas de la ocupación teotihuacana para entender así los procesos que se involucrarían en el desarrollo del estado, así como para aplicar diferentes técnicas geofísicas (Manzanilla, 1994a,1994b,1996). Paralelamente, la intervención realizada por el INAH permitió ampliar los trabajos iniciados por Basante, Munera y Soruco en la excavación de la cueva astronómica determinando que dicha cueva se complementa con otras dos, definiendo un complejo subterráneo ceremonial de la época clásica con una reocupación Coyotlatelco tardío-Mazapa (Basante, 1986; Moragas, 1995). Los años subsiguientes permitieron seguir avanzando en la tipología de las cerámicas de la fase Coyotlatelco y Mazapa, y por otro lado 


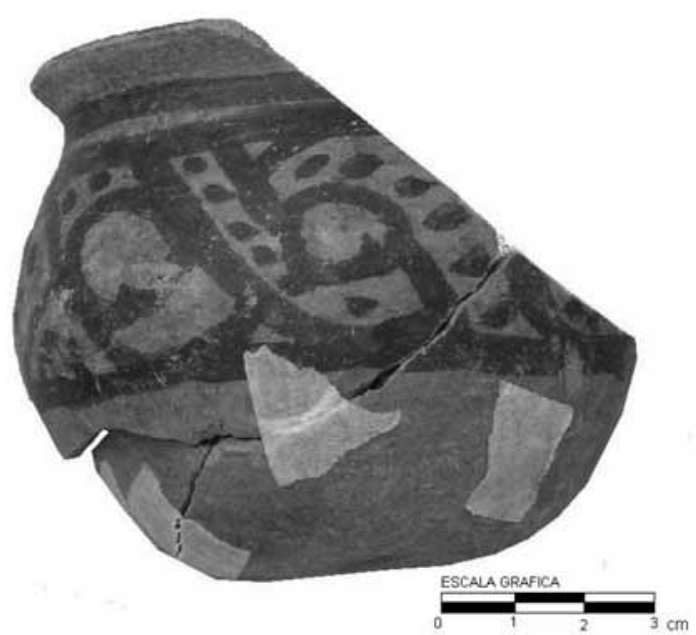

Figura 2. Cerámica Coyotlateco (foto Luis Gamboa).

empezar a replantearse algunas de las cuestiones vinculadas a los cambios y transformaciones culturales inmediatas a la caída de la ciudad (Moragas, 1995; López, 2003; Nicolás, 2003). Asimismo, en estos años Luis Gamboa excava la periferia de la ciudad en donde se muestra la presencia temprana de estas cerámicas coyotlatelcoas sobre pisos de fases clásicas sin evidencias de abandono o discontinuidad (Gamboa, 1998). Para estas fechas se publican nuevos datos acerca de la cronología de lo Posclásico en la cuenca de México que indican que se debe hacer una revisión de la secuencia cronotipológica que suponen un cambio en el modelo explicativo secuencial de Metepec a Azteca III y que muestra contemporaneidades y discontinuidades fuera de modelos culturales (Parsons y otros, 1996). Todo ello supuso un revulsivo en la concepción de la secuencia del colapso en Teotihuacan, así como del papel de sus supuestos "invasores". La bipolarización del discurso acerca de los factores que conllevaron al colapso de la cultura teotihuacana se complicaba con las evidencias de la presencia temprana de coyotlatelcos antes de la fecha mítica del 750 d.C. Es por ello que la discusión hasta esos momentos se había centrado en considerar a los coyotlatelcos como los causantes directos o indirectos de estas crisis y su implicación con los factores externos (crisis climáticas) o factores internos (crisis sociales e ideológicas). Lo cierto es que en los últimos años no se han presentado nuevas propuestas sobre el colapso de la cultura teotihuacana. La reciente publicación de Gazzola mantiene el modelo tradicional del colapso, bajo el conflicto vertical de las élites versus no élites y el descontento social ${ }^{2}$ (Gazzola, 2009).

\section{Añadiendo nuevos factores a la discusión del colapso de Teotihuacan. Procesos y eventos}

Las diferentes explicaciones no terminan de conceptualizar un marco claro del colapso de Teotihuacan sobre todo en el momento en que se intenta aglutinar bajo un único modelo explicativo que parte de los indicadores arqueológicos más visibles: el incendio, el saqueo y el cambio de la cultura material. Sin embargo, creo que el colapso es más comprensible si añadimos otros factores que complementan el escenario histórico y definimos lo que realmente significan los indicadores arqueológicos que tomamos como base de dicho argumento. Es innegable que la visibilidad arqueológica del incendio, el saqueo y el cambio de la cultura material marcan mucho las interpretaciones, pero hemos de observar también con cuidado los procesos por los cuales se llegan a estas evidencias. No es tanto conseguir una secuenciación de los factores, sino la integración de estos en un escenario en donde se incorporen los diferentes actores que intervienen en los años previos y durante el colapso. Es por ello que considero que hay que distinguir los eventos, fenómenos rápidos en el registro arqueológico y los procesos, tal vez menos evidentes pero más largos en el tiempo y que contribuyen a los anteriores. Es decir, no podemos llegar a los eventos sin tener en cuenta los procesos históricos arqueológicamente menos visibles.

\section{Algunas propuestas de indicadores de procesos históricos}

La sociedad del Xolalpan tardío y Metepec muestran ya evidencias de problemáticas no resueltas en la sociedad teotihuacana que suponen, por un lado, el despoblamiento en la periferia y la reocupación coyotlatelco en tiempos clásicos (Gamboa, 1998) como una mayor densidad de poblamiento en el centro con la ocupación de las calles por asentamientos adosados y el cierre de los barrios étnicos. Pueden ser interpretados como un aumento de la población o en cambio por un reagrupamiento de la misma en una dinámica distinta ${ }^{3}$. A nivel regional hemos de considerar la presencia coyotlatelca temprana en el valle de México, los asentamientos teotihuacanos en el valle de Toluca, 
la disminución de la influencia teotihuacana en la ruta del Anaranjado temprano (García Chávez, 1998; González de la Vara, 1999; Parsons y otros, 1993, 1996; Rattray, 1998; Sugiura, 1996). También se detecta para la fase Metepec cierto desabastecimiento en algunos productos vinculados a las ofrendas y ajuares funerarios (Sempowski, 1992, 1999; Sempowski y Spence, 1994). Caso interesante es la presencia temprana de asentamientos coyotlatelcos en el sur de la cuenca de México en fechas en las que supuestamente el poder de Teotihuacan sigue estando presente y cuando menos no perciben a los coyotlatelcos como una amenaza en el territorio inmediato. Interpretarlo como una evidencia de debilidad de la capital o una incorporación más dentro de una sociedad multiétnica es ya una cuestión de las propuestas.

No obstante, queremos incorporar otros elementos a la discusión del colapso que comprenden procesos graduales en el tiempo. Uno de ellos es la crisis del modelo de ciudad teotihuacana que involucra la desacralización, deterioro y abandono parcial de la ciudad. No podemos obviar que la cultura teotihuacana está profundamente marcada por la propia capital y la concentración de la población en la misma. Asimismo, hay que recordar que la ciudad es la representación cosmológica y política de un modelo muy particular de gobierno de la misma. Desde la multiplicidad de factores que implica la fundación de una ciudad y su mantenimiento también hemos de considerar todos aquellos que conjugan en el final de la misma ${ }^{4}$. Resulta sugestivo el análisis de Graulich que analiza el mito del Quinto Sol y que aunque sean reinterpretaciones del Postclásico, las fechas no dejan de resultar sugestivas ${ }^{5}$ (Graulich, 1990: 90-92).

En el proceso de abandono progresivo de la ciudad hemos de destacar la desmantelación y las ofrendas matadas que se localizan en lugares diversos de la ciudad. Se observan cambios sustanciales en la Calzada de los Muertos y la Ciudadela así como la ruptura sistemática de esculturas en este lugar y Xalla. Incensarios tipo teatros desbaratados y redepositados intencionalmente se han encontrado en Oztoyohualco y Tlamimilolpa (Linné, 1942: 172; Manzanilla, 1993). En ambos casos parece que nos encontramos con un ritual establecido alrededor de un contexto doméstico funerario. En cambio, no tenemos constancia de incendios generalizados de la ciudad hasta el colapso de la misma. El abandono progresivo de las estructuras es otro de los procesos que nos indican la ruptura de la organización interna de la ciudad. Las excavaciones de la Ventilla $\mathrm{C}$ muestran inundaciones en el gran espacio abierto que sugieren falta de mantenimiento de las grandes estructuras (Gómez y Núñez, 1999:112).Una mención cabe hacerse sobre el cambio de las técnicas constructivas y localidad de los edificios atribuidos a los coyotlatelcos, lo que sugiere también la pérdida del savoir-faire y de la organización el trabajo especializado involucrado en la construcción y diseño de los grandes edificios.

Los eventos resultan de la presencia de los incendios concentrados en el centro ceremonial de la ciudad vinculada principalmente a los edificios del poder religioso y político de la ciudad, relacionados con el ejercicio del poder en Teotihuacan. El incendio y los saqueos resultan muy visibles arqueológicamente y por lo tanto marcan la interpretación con hechos violentos, pero aún no podemos determinar si fueron fuegos rápidos o lentos. Es decir, si son resultados de un ataque o de una ritualización del cierre vinculado a la desacralización del edificio. Sin duda alguna los coyotlatelcos que reocuparán sectores de la ciudad contribuirán a una reinvención de la misma en la que aparentemente el área de la Pirámide del Sol es significativa tanto por la reocupación de las cuevas situadas al este y sudeste de las mismas así como de la construcción de los edificios alrededor de la Pirámide del Sol y los edificios anexados en la Plaza de la Pirámide de la Luna o la estructura 1B de la Ciudadela ${ }^{6}$ (Cabrera Castro, 1990; Manzanilla y Arrellín, 1999; Moragas, 1995; Matos, 1995, 1999).

\section{La transición del Clásico al Epiclásico en Teotihuacan. Demasiadas preguntas y pocas respuestas}

En términos generales, la transición del Clásico al Epiclásico representa una escisión del control central de los grandes centros urbanos del Clásico y, en consecuencia, pone al descubierto los conflictos entre facciones que, libres del marco de sujeción impuesto por el Estado clásico, han de desarrollar sus propios marcos de acción política y social. Aún nos queda mucho por averiguar acerca de la sociedad del Epiclásico en Teotihuacan y el tipo de organización política que se genera en este territorio dominado anteriormente por una gran metrópolis con un complejo material homogéneo y de distribución centralizada $^{7}$. En este modelo hemos de entender 
que en un mismo escenario nos encontramos con dos sociedades culturalmente distintas y en dos momentos de desarrollo sociocultural distinto: los teotihuacanos en un rápido proceso de descomposición política y los coyotlatelcos que se encuentran en un proceso de asentamiento territorial en el área que conformaba la capital y la periferia inmediata, bajo el dominio de la ciudad. Es posible que sean dos tradiciones culturales distintas, pero no implica necesariamente que sean excluyentes en un primer momento. Aquí vamos a introducir algunas de las cuestiones que son pertinentes a la hora de considerar la sociedad epiclásica en Teotihuacan y las problemáticas de identificar a los diferentes grupos desde la arqueología y las fuentes históricas.

En primer lugar, los movimientos poblacionales en Mesoamérica y su relación con Teotihuacan. Nuestros protagonistas principales son los coyotlatelcos, pueblo de origen no identificado aún pero que mayoritariamente se consideran de alguna zona del Bají $^{8}$. Sin duda alguna los coyotlatelcos son uno de los indicadores clave en la conformación de dicho período, pero no son los únicos ${ }^{9}$. Hay que considerar los movimientos de grupos teotihuacanos que salen de la ciudad y que podemos identificar con la llegada de nuevas gentes al valle de Toluca desde la fase Tlamimilolpan hasta Metepec. La diferencia es que si bien no afectan a la metrópolis en su momento de esplendor, sí que podrían ser lugar de acogida para las fases tardías del clásico ${ }^{10}$.

Otras propuestas sugieren asociar a los teotihuacanos de final del Clásico a los Olmeca-Xicallangas mencionados en las obras del los cronistas como fray Bernardino de Sahagún, Domingo Chimalpahin, Fernando del Alva Ixtlilxochitl, Diego Muñoz Camargo, el códice Matritense, por citar algunos ejemplos. Estos estarían vinculados a la fundación de Cacaxtla, la conquista de Cholula. Sin embargo otros autores como Chadwick sugieren que estos Olmeca-Xicallangas son descendientes de teotihuacanos de origen mixteco y chochopopoloca ${ }^{11}$ (Chadwick, 1966: 8-9). Rivas Castro, López Austin y López Luján sin embargo consideran que en los Olmeca-Xicallangas debe verse a un grupo compuesto por diversas entidades étnicas, en los que cabe abarcar a grupos del tronco lingüístico otomange, a mixtecos, a mazatecos, chochopopolocas y a gentes de procedencia náhuatl (López Austin y López Luján, 1996: 166; Vargas, 1998). En esta ecuación de pueblos también se hacen presentes los otomíes como los causantes de la caída teotihuacana, tesis defendida por Jiménez Moreno ${ }^{12}$. Sin embargo para Piña Chan los otomíes que usaban cerámica coyotlatelco, convivieron con los teotihuacanos y a su caída desarrollarían otros estilos locales (Piña Chan, 1975: 543).

Dicha variabilidad sería reflejo, entre otros muchos factores, de los movimientos poblacionales de la cuenca ya a fines del clásico y la visibilización de otras identidades regionales después de la pérdida del monopolio cultural y económico de Teotihuacan. En todo caso hemos de considerar que hay dos escenarios del colapso: el que se determina en el centro de la ciudad y el que sucede en la periferia inmediata. En el centro urbano de Teotihuacan lo más destacable no es la construcción ex novo de la que se tienen pocos ejemplos, sino la remodelación del espacio interior que se observa en las construcciones clásicas teotihuacanas. No sabemos exactamente por qué se realiza esto. Más evidente resulta cuando la remodelación del espacio se refiere a una modificación de la viabilidad interna de todo un conjunto, normalmente a consecuencia del cierre de varios pasillos. Ello puede relacionarse con la organización del reasentamiento que implica ideas como redistribución del espacio a consecuencia de las nuevas actividades que se realicen dentro. Si las antiguas construcciones dedicadas a actividades de tipo administrativo-ceremonial son sedes de familias o grupos familiares, es de esperar que se desarrollen actividades de tipo doméstico y artesanal. La remodelación del espacio puede deberse a la necesidad de readaptar el espacio a estas nuevas actividades. Los cambios en la viabilidad interna pueden interpretarse como una manera de reorganización de las unidades residenciales para el control de los movimientos de gentes ajenas a ese conjunto o como en una delimitación espacial de los diferentes grupos y actividades que se realizan dentro. Resumiendo, los cambios en la viabilidad interior pueden responder a cambios en la funcionalidad de los cuartos, al acomodo de los diversos subgrupos familiares y a cuestiones defensivas.

La ciudad pierde su aspecto de globalidad. Durante el Clásico la ciudad fue concebida como un todo en el que, bajo el gobierno de unas élites, nada se deja al azar. Los coyotlatelcos reocuparán partes de la ciudad rompiendo esa globalidad en la que fue diseñada para otros usos, aunque no se aprecian grandes obras de construcción o en todo caso que siguen utilizando las infraestructuras anteriores. Este es el gran problema para determinar 
con mayor claridad la presencia coyotlatelca y el uso de la ciudad por los mismos, ya que a menudo utilizan las propias estructuras teotihuacanas remodelando sus espacios ${ }^{13}$. Otra de las grandes dudas es visibilizar una élite coyotlatelca propia, ya que posiblemente no habría la necesidad de crear un proyecto ideológico de gran alcance como lo hicieron los teotihuacanos. Lo mismo sucede para identificar elementos de élite en la cultura material que sugieran la presencia de artesanos especializados en objetos de lujo, como nos acostumbraban los teotihuacanos. Lo que implica también cambios substanciales en los circuitos comerciales bajo las nuevas relaciones del poder. La presencia de malacates en Teotihuacan no se encuentra hasta el período Epiclásico (Smith y Hirth, 1988). Durante el Epiclásico la situación sociopolítica permitió el desarrollo de mercados regionales en las que las élites rurales del valle de México demandan piezas textiles, sobre todo ichcahuipilli, y en las que la producción de algodón se hace más evidente arqueológicamente por la presencia de malacates, agujas de coser y ciertos tipos cerámicos ${ }^{14}$. En hacienda Metepec la producción de las puntas San Marcos excede la propia consumición interna, y Rattray propone que es un producto de intercambio entre los habitantes de hacienda Metepec y otros asentamientos. En Oxtoticpac se determina una explotación de las minas de tezontle y un taller de cerámica (García Chávez, 1995: 126; Rattray, 1981: 215). Rattray identifica varios talleres de cerámica coyotlatelco con cierta especialización en un tipo determinado (Rattray, 1981: 215-216). Tal vez las zonas de Maquixco, la zona de la parroquia de San Juan de Teotihuacan y Puxtla proporcionaron excedentes agrícolas para el comercio, a pesar de que faltan mayores estudios para probar esta hipótesis. Finalmente, la zona del norte del valle de Teotihuacan, con sus cuevas y oquedades, pudo fungir como lugar de almacenamiento de excedentes dotando de esta manera a sus habitantes de una posibilidad de manejar excedentes para el intercambio.

Raúl García Chávez estableció la tipología de la cerámica coyotlatelco en el valle de Teotihuacan, identificando complejos regionales y asociando a estos a las tres unidades políticas diferentes estimadas por el Teotihuacan Basin Project (Figura 3) (García Chávez, 1995). Si las evidencias que menciona García Chávez son correctas, la ocupación de los asentamientos del Clásico como Azcapotzalco, Xico, cerro
Portezuelo, cerro de la Estrella y Teotihuacan muestra una ocupación por gente de tradición teotihuacana y por la gente que producía la cerámica coyotlatelco (García Chávez, 1995: 292). Ello nos indica que si existió una convivencia temporal entre gentes de tradición teotihuacana clásica con las gentes que utilizaban principalmente cerámica coyotlatelco, esta tenía un carácter básicamente pacífico y que determinó en un primer momento un panorama político caracterizado por el desplazamiento de la población residente en la urbe teotihuacana. En ese momento debieron de establecerse las nuevas alianzas políticas, étnicas y territoriales.

Tampoco sabemos mucho de las creencias particulares de los coyotlatelcos. El entorno de estos cultos viene determinado por la ocupación en cuevas, las estructuras encontradas en la plataforma en "U" de la Pirámide del Sol y la existencia de determinados elementos en la zona de la Pirámide de la Luna, que sugieren que la ocupación en esta zona tuvo unas características diferentes a la reocupación de los espacios en los conjuntos departamentales de Tetitla, Zacuala, Yayahuala, la Ventilla C. En la cueva de las Varillas se encontró una estructura bajo el agujero del techo de la cámara sepulcral que debía de tener un chorro de agua importante en el momento de llover ${ }^{15}$. Bajo esta estructura se encontraron varios entierros de neonatos, dispuestos en una orientación este-oeste rodeando la forma de esta estructura (Manzanilla, 1994a: 59). La datación de este conjunto se fecha en Coyotlatelco tardío (Manzanilla y Arrellín, 1999: 429-431). No obstante hay evidencias de este culto en fases posteriores a la de Coyotlatelco, según las interpretaciones de Jarquín y Martínez, de las ofrendas de vajillas yuxtapuestas y la escultura de Xipe Totec, el dios desollado, de la procreación de la tierra, lo que sugiere que la pervivencia de este culto, con variaciones, se desarrolló a lo largo de todo el período prehispánico en la zona teotihuacana (Jarquín y Martínez, 1991). La reocupación o al menos la presencia de materiales coyotlatelcos en áreas de marcado carácter religioso, como son la zona de la Pirámide del Sol y de la Luna y el conjunto de La Ciudadela, nos muestra una cierta actividad ritual tal vez más relacionada con ideas de tradición y cosmovisión que por la representación simbólica del Estado teotihuacano. Otros elementos rituales a considerar son la presencia de nuevos tipos de formas específicas para los rituales, como son 


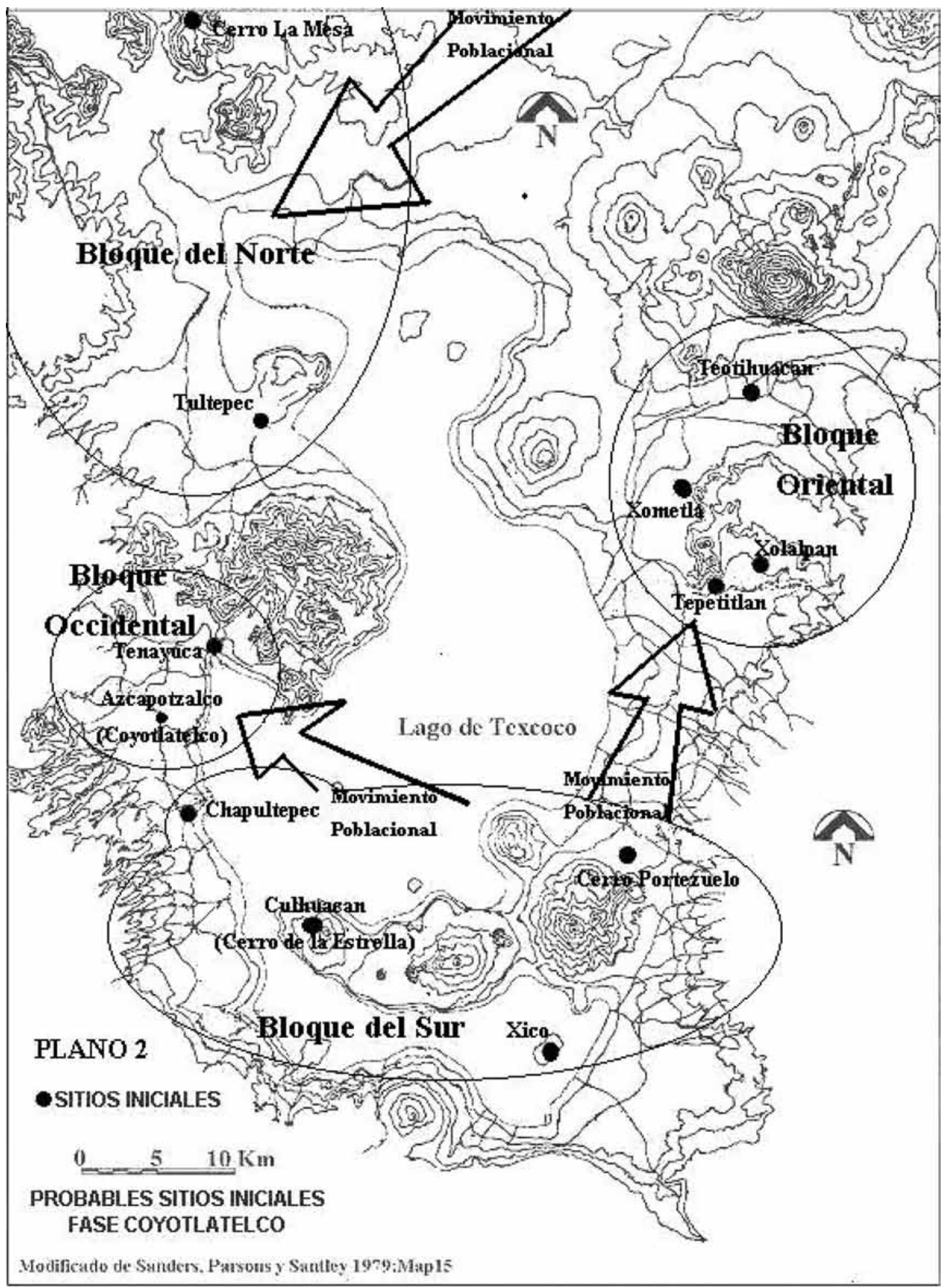

Figura 3. Complejos regionales de la cerámica Coyotlatelco (García Chávez, 1995). 
los sahumadores y cucharones que sugieren nuevos rituales en los que el copal y el pulque aparecen mencionados (García Chávez y otros, 1998b: 78). Los excéntricos trilobulados parecen mantenerse en este período aunque en mi opinión hay que tener en cuenta que los contextos aparecen mezclados, por lo que la continuidad de este elemento no es tan clara (Stocker y Spence, 1973: 195-198). Barbour considera que se pueden detectar dos tradiciones diferentes para las figurillas teotihuacanas a fines de Xolalpan y que perdurará hasta el Postclásico, lo que vincula como indicadores de cambios políticos en la ciudad (Barbour, 1998: 253).

Los sacrificios humanos siguen con evidencias de decapitaciones y del uso de cráneos humanos como recipientes. Calotas del mismo tiempo y evidencias de decapitación se hallan en materiales aparecidos en la zona de la Pirámide de la Luna y las cuevas al sudeste de la Pirámide del Sol (Moragas, 1995). La presencia de perros asociados a entierros perdura. En la Cueva III se encontró el cráneo de un perro directamente asociado a un entierro secundario, indirecto de un individuo masculino (Moragas, 1995: 50). Lo mismo indica Manzanilla para la Cueva del Pirul, en la que determina dos esqueletos de perro y un esqueleto de lobo, relacionados con diversos entierros. Esta cueva tiene una cronología posteotihuacanas con contextos coyotlatelcos, mazapas y aztecas ${ }^{16}$ (Manzanilla, 1994a: 59; Manzanilla y Arrellín, 1999: 431y 448).

\section{Conclusiones: (Re) Construyendo la historia de Teotihuacan}

Quedan aún muchos temas por analizar para poder construir la historia de Teotihuacan. Por un lado analizar con más cuidado los fenómenos de resiliencia y de procrastinación que se presentan en ambas sociedades, considerar la reinvención y readaptación del espacio urbano (la ciudad) y la periferia (el campo) o analizar con mayor detalle el nivel de complejidad sociopolítica de los coyotlatelcos.

Las sociedades en transición son períodos complejos, interesantes y significativos, pero mucho más difíciles de caracterizar arqueológicamente por las presencias y ausencias de determinados materiales. En algunos casos parece que solo se puede caracterizar este período por lo que se pierde culturalmente del período precedente. Debemos implementar una metodología propia que considere la velocidad del cambio en las sociedades involucradas y su reflejo en la cultura material.

Teotihuacan es un estudio de caso, por lo tanto paradigmático para el estudio del colapso en las sociedades complejas.

\section{Referencias Citadas}

Acosta, Jorge R.

1964 El Palacio del Quetzalpapalotl. Memorias INAH no 10. México.

Basante, Oscar

1986 Ocupación en cuevas en Teotihuacan.Tesis de Licenciatura ENAH. México.

Barbour, Warren

1998 "The Figurine Chronology of Teotihuacan, Mexico".

Brambila, Rosa y Cabrera, Rubén (coords) Los ritmos de cambio en Teotihuacan: reflexiones y discusiones de su cronología. Colección Científica INAH No 366 México, pp. 243-253.

Batres, Leopoldo

1889 "Teotihuacan o la ciudad sagrada de los toltecas". Talleres de la escuela nacional de artes y oficios (monografías de arqueología mexicana), México.

Braniff, Beatriz

1972 "Secuencias arqueológicas en Guanajuato y el Centro de México, intento de correlación”. Teotihuacan: XI Mesa Redonda de la SMA: México, pp. 273-319.

Cabrera Castro, Rubén

1990 "El Proyecto Arqueológico Teotihuacan 80-82. Resumen de sus resultados en la Ciudadela". A. Cardós de Méndez (cood.) La época Clásica: Nuevos hallazgos, nuevas ideas: MNA, INAH México, pp. 73-80.

Chadwick, Robert

1966 "The Olmeca-Xicallanga of Teotihuacan: A preliminary Study “. Mesoamerican Notes 7-8, pp. 1-23.

Chavero, Alfredo

1987 "Historia antigua y de la conquista de México", México a través de los siglos, vol. Dos, México, Editorial Cumbre, pp. 106-110.

Carballo, David M.

2009 "Household and Status in Formative Central Mexico: Domestic Structures, Assemblages, and Practices at La Laguna, Tlaxcala". Latin American Antiquity 20 (3), pp. 473-501.

Carballo, David M. y Thomas Pluckhahn

2007)" Transportation Corridors and Political Evolution in Highland Mesoamerica: Settlement Analyses Incorporating GIS for Northern Tlaxcala", Mexico. Journal of Anthropological Archaeology 26 (4) pp. 607-629.

Cohodas, Marvin

1989 "The Epiclassic Problem: A review and Alternative Model”. Diehl (edit). Mesoamerican after the decline of 
Teotihuacan AD700-900. Dumbarton Oaks. Washington, pp. 219-239.

Cowgill,George

2008 “An Update on Teotihuacan”. Antiquity 82, pp. 962-975.

Cook M.

1947 "The interrelation of population, food supply, and building Preconquest Central Mexico". American Antiquity 8: 45-52.

García Chávez, Raúl

1995 Variabilidad Cerámica en la Cuenca de México durante el Epiclásico. Tesis de Maestría ENAH, México.

Gamboa Cabezas, Luis Manuel

1998 La distribución de la cerámica de fase coyotlatelco en el valle de Teotihuacan. Tesis de Licenciatura ENAH-INAH, México.

García Chávez, Raúl; Felipe Ramírez; Lorena Gámez y Luis Córdoba

1998 Chimalhuacan: Rescate de una Historia. Municipio de Chimalhuacan, INAH, México.

Gazzola, Julie

2009 "Una propuesta sobre el proceso, factores y condiciones del colapso de Teotihuacán”, en Dimensión Antropológica, INAH, No 31, año 2009 (Ver http://www.dimensionantropologica.inah.gob.mx).

Graulich, Michel

1990 Mitos y rituales del México Antiguo. Colegio Universitario de ediciones Itsmo 8, Madrid.

Gómez Chávez, Sergio Jaime

1999 "Análisis preliminar del patráon y la distribución de entierros en el barrio de la Ventilla", en Manzanilla, Linda y Serrano, Carlos (ed.). Prácticas Funerarias en la Ciudad de los Dioses. Los enterramientos humanos de la antigua Teotihuacan: IIA-UNAM-DGAPA, México, pp. 81-147.

González de la Vara, Fernán

1999 El Valle de Toluca hasta la caída de Teotihuacan. Colección Científica INAH: 389, México.

Jarquín Pacheco, Ana María y Enrique Martínez Vargas, 1991 "Sacrificio de niños. Una ofrenda a la deidad de la lluvia en Teotihuacan". Arqueología 6: 69-85, INAH, segunda época, México.

Jiménez Moreno, Wigberto

1966 "Mesoamerica before the Toltecs". Paddock, John (ed.) Ancient Oaxaca. Discoveries in Mexican Archaeology and History: Stanford University Press, Stanford, California. pp. 1-83.

Linné, Sidwald

1942 Mexican Highland Cultures. Archaeological Researches at Teotihuacan, Calpulalpan and Chalchicomula in 1934-35. Ethnographical Museum of Sweden, New Series, Publication $\mathrm{N}^{\mathrm{o}}$ 7, Stockholm.

Litvak King, Jaime

1970 "Xochicalco en la Caída del Clásico: Una hipótesis". Anales de Antropología: UNAM, pp. 131-144.

Litvak King, Jaime

1978 "Central Mexico as a Part of the General Mesoamerican Communications System”. Lee, Thomas Navarrete, Carlos (eds) Mesoamerican Communication Routes and Cultural Contacts: 115-122. Papers of NewWorld Archaeological Foundation $\mathrm{N}^{\circ} 40$, Utah.
López Austin, Alfredo y Leonardo López Luján 1996 El Pasado Indígena. El Colegio de México. Fideicomiso Historia de las Américas, serie Ensayos. FCE, México.

López, Claudia; Claudia Nicolás y Linda Manzanilla 2006 "Atributos morfológicos y estilísticos de la cerámica Coyotlatelco en el centro ceremonial de Teotihuacan", El fenómeno Coyotlatelco en el Centro de México: Tiempo, Espacio y Significado, Laura Solar (ed.), INAH, México, pp. 215-230.

Lorenzo, José Luis

1968 "Clima y Agricultura en Teotihuacan". J.L. Lorenzo (ed.) Materiales para la arqueología de Teotihuacan: 53-72, Serie investigaciones 17, INAH, México.

Manzanilla Naim, Linda

1993 "Los conjuntos residenciales teotihuacanos". Manzanilla, Linda (coord.). Anatomía de un conjunto residencial teotihuacano en Oztoyohualco: vol. I-Las excavaciones, IIA-UNAM, México, pp. 31-41.

1994a "Geografía Sagrada e Inframundo en Teotihuacan". Antropológicas No 11, Nueva Época: 53-66, UNAM.

1994b "Caves and Geophysics: An Approximation to the Underworld at Teotihuacan, México". Archaeometry, vol. 36, $\mathrm{N}^{\circ}$ 1, Oxford University, London.

1994c "Las cuevas en el mundo mesoamericano". Ciencias No 36: 59-66. Facultad de Ciencias-UNAM. México.

2001 "Agrupamientos sociales y gobierno en Teotihuacan, Centro de México", en: Reconstruyendo la ciudad maya: el urbanismo en las sociedades antiguas, Andrés Ciudad Ruiz, Ma. Josefa Iglesias Ponce de León y Ma. del Carmen Martínez Martínez (eds.), Publicaciones de la Sociedad Española de Estudios Mayas No 6. Madrid, pp. 461-482. 2006 "Estados corporativos arcaicos. Organizaciones de excepción en escenarios excluyentes", Revista Cuicuilco vol. 13, No 36 (enero-abril), ENAH: 13-45.

Manzanilla, Linda y Leonardo López Luján

2001 "Exploraciones en un posible palacio de Teotihuacan: el Proyecto Xalla (2000).

Manzanilla, Linda, Claudia López y Claudia Nicolás

2006 "La cerámica de la cuenca de México durante el Epiclásico/transición al Posclásico temprano (650900 d.C.)", en: La producción alfarera en el México antiguo, vol. III (La alfarería del Clásico tardío [700-1200 d.C.]), Beatriz Leonor Merino Carrión y Ángel García Cook (eds.), Colección Científica, Instituto Nacional de Antropología e Historia: 169-186 (entregado en junio del 2000; ISBN 968-03-0144-3).

Manzanilla Naim, Linda, Arrellin, Rocío

1999 "Los entierros de los túneles al este de la Pirámide del Sol: Proyecto UNAM 1987-1996". Manzanilla, Linda y Serrano, Carlos (eds.), Prácticas Funerarias en la Ciudad de los Dioses. Los enterramientos humanos de la antigua Teotihuacan. IIA-UNAM-DGAPA, México, pp. 427-458.

Matos Moctezuma, Eduardo

1990 Teotihuacan.La Metrópoli de los Dioses. Corpus Precolombino. Sección Las Civilizaciones Mesoamericanas. Jaca Book Spa Milano.

Matos Moctezuma, Eduardo

1995 La Pirámide del Sol, Teotihuacan. Antología, Artes de México para el Instituto Cultural Domecq, INAH, México. 
Matos Moctezuma, Eduardo

1996 "Surgimiento y caída de Teotihuacan: dos hipótesis". Mastache, Alba Guadalupe y otros (coords.), Arqueología Mesoamericana. Homenaje a W. T. Sanders. vol. I: 210212, INAH y Arqueología Mexicana.

Matos Moctezuma, Eduardo

1999 "Teotihuacan". Dossiers de l'Archéologie, $\mathrm{N}^{\circ} 245$ : 82-89, juillet-auot 1999.

Messenger, Lewis C.

1990) "Ancient Winds of Change: Climatic settings and prehistoric social complexity in Mesoamerica". Ancient Mesoamerica 1: 21-40, Cambridge University Press, USA.

Millon, René

1973 Urbanization at Teotihuacan, México. Vol. 1, The Teotihuacan Map Text. University

Mooser, F.

1968 "Geología, naturaleza y desarrollo del valle de Teotihuacan”. JL Lorenzo (ed.), Materiales para la arqueología de Teotihuacan: 29-37. Serie investigaciones 17, INAH, México of Texas Press. Austin.

Moragas Segura, Natàlia

1995 Aportaciones cronológicas y ceremoniales en dos cuevas al sudeste de la Pirámide del Sol, Teotihuacan, México. Tesis de Licenciatura. Universitat de Barcelona.

Palerm, Ángel y Eric Wolf

1972 Agricultura y Civilización en Mesoamérica. Editorial Gernika, México.

Parsons, Jeffrey; Elisabeth Brumfield y Mary Hodge 1996 "Developmental implications of earlier dates for early aztec in the Basin of Mexico". Ancient Mesoamerica 7: 217-230. Cambridge University Press, USA.

Piña Chan, Román

1975a Teotenango: El antiguo lugar de la Muralla. Memoria de las excavaciones arqueológicas, tomo I-II. Dirección de Turismo, Gobierno del Estado de México, México.

Rattray, Evelyn Ch.

1981c "La industria de la obsidiana durante el Período Coyotlatelco". Revista Mexicana de Estudios Antropológicos XXVII: 2: 213-223, México.

1998 "Fechamientos por Radiocarbono de Teotihuacan". Brambila, Rosa, Cabrera, Rubén (coords.), Los ritmos de cambio en Teotihuacan: reflexiones y discusiones de su cronología. Colección Científica INAH N ${ }^{\circ} 366$ : 255-281, México.
Sanders, William T.

1965 The cultural Ecology of the Teotihuacan Valley. Departament of Sociology and Anthropology, Pennsylvania State University, University Park, Pennsylvania.

Sanders, William T. y Barbara Price

1968 Mesoamerica: The Evolution of a Civilization. Random House, New York.

Sempowski, Martha

1992 "Economic and Social Implications of Variations in Mortuary Practices at Teotihuacan”. Berlo, J. (ed.) Art, Ideology and the City of Teotihuacan. 27-57 Dumbarton Oaks.

Sempowski, Martha y Michael Spence

1994 Mortuary practices and skeletal remains at Teotihuacan.

University of Utah, Salt Lake City.

Sempowski, Martha

1999 "The potential role of human interment in household ritual at Tetitla". Manzanilla, Linda y Serrano, Carlos (eds.). Prácticas Funerarias en la Ciudad de los Dioses. Los enterramientos humanos de la antigua Teotihuacan. 473-499, IIA-UNAM-DGAPA, México.

Siebe, Claus; Michael Abrams; José Luis Macías y Johannes Obenholzner

1996 "Repeated volcanic disasters in Prehispanic time at Popocatepetl central Mexico: Past key to the future? Geology, vol. 24, № 5: 399-402.

Smith, Michael y Kenneth Hirth, G.

1988 "The development of prehispanic cotton -spinning technology in Western Morelos". Journal of Field Archaeology: 15: 349-358.

Stocker, Terrance; L. Michael W. Spence

1973 "Trilobal eccentrics at Teotihuacan and Tula". American Antiquity: 195-198, vol. 38, № 2 .

Sugiura Yamamoto, Yoko

1996 "El Epiclásico y el problema del Coyotlatelco vistos desde el valle de Toluca". Mastache, Alba Guadalupe y otros (coords.) Arqueología Mesoamericana. Homenaje $a$ W. T. Sanders. vol. I: 233-255, INAH y Arqueología Mexicana.

Webb, Malcolm

1978 "The Significance of the "Epiclassic" Period in Mesoamerican Prehistory". Browman, L Cultural Continuity in Mesoamerica: 155-178. Mouton Publishers, The Hague, Paris.

\section{Notas}

1 "Una característica que singulariza a Teotihuacan frente a muchas sociedades contemporáneas y posteriores son las viviendas multifamiliares. Cada una de las diversas familias relacionadas entre sí por parentesco, actividades comunes y relaciones de afinidad, tienen un grupo de cuartos, pórticos y patios para vivir y reproducirse" (Manzanilla, 2006: 20).

2 Sospechamos que entre los años 600 y 650 d.C., la falta de mecanismos de regulación eficientes que enfrentaran el descontento social generalizado habría provocado el éxodo y finalmente el incendio tanto en edificios públicos como de templos que paulatinamente se habrían convertido en ruinas para luego ser desmantelados y saqueados de sus ofrendas fundacionales, mismas que serían utilizadas para acompañar las nuevas migraciones (Gazzola, 2009).
3 En épocas históricas de crisis se han observado movimientos de concentración de grupos familiares como una estrategia de respuesta.

4 En la antigüedad la constitución de la ciudad aparece a menudo referida a un pacto entre hombres y dioses y que se relaciona con algún hecho sangriento. Los mitos y leyendas fundacionales recurren a menudo a este elemento. La ciudad se presenta, por lo tanto, como un lugar seguro por un lado, en donde la erección de un templo o estructura arquitectónica se hace bajo unas determinadas normas y con una serie de actores. Pero, por otro lado, no queda desvinculada de un pasado mítico-histórico en el que se menciona a algún hecho de naturaleza sangrienta y caótica. Aunque no sabemos cuáles eran los mitos y ritos de fundación propiamente 
teotihuacanos, sí sabemos que muy probablemente tenían su origen en la zona norte de la ciudad y en la que las cuevas tuvieron un papel primordial.

5 En el Origen de los Mexicanos y la Relación de la Genealogía se formulan las fechas de 712 y 765 de nuestra era, Chimalpahin ofrece la de 670, Motolínea detalla que el Cuarto Sol pereció en el 694 y el Quinto Sol nació en el 719. Finalmente, los Anales de Cuauhtitlan el 751 (Graulich, 1990: 90-92).

6 Sobre este último ejemplo hay que recordar que proceden de excavaciones antiguas y que se mencionan solo como posteotihuacanos.

7 Se han determinado varios escenarios históricos mesoamericanos para el Epiclásico que son: El centro de México con especial importancia en la cuenca de México, Tula y Cholula; la expansión tarasca en Michoacán: Oaxaca y los conflictos con los señoríos mixtecos; el norte de Yucatán; la expansión de quichés, cakchiqueles y rabinales en los Altos de Guatemala. En todos ellos se interpretan conflictos entre grupos de tradición clásica y otros grupos que encabezaban nuevas tendencias (López Austin y López Luján, 1999: 38).

8 La muestra de entierros es pequeña, pero es la única excavada de manera extensiva para tiempos Coyotlatelco y Mazapa en el valle de Teotihuacan. La riqueza de la información de las áreas de actividad asociadas, los fechamientos, los restos botánicos y faunísticos permiten conocer mejor las prácticas funerarias de los grupos que llegaron al valle a raíz de la caída de la ciudad del Clásico. Por los estudios genéticos podemos suponer que sus orígenes eran diversos, pero no del valle. Pudieron haber llegado poco antes de colapsarse el sistema del Clásico, incluso participar del caos. También se pudieron haber casado con mujeres de la región, como lo sugiere el estudio de isótopos de estroncio. Así, nos inclinamos hacia la hipótesis de que grupos del Bajío y centro-norte de México pudieron haber llegado en oleadas al centro de México hacia el siglo sexto de la era cristiana (Manzanilla y Arrelín, 1999: 451)".

9 Las propuestas van desde considerar a los coyotlatelcos como los descendientes de poblaciones Metepec, inmigrantes que desplazan a los pobladores clásicos y/o intrusos que ocuparon un Teotihuacan abandonado (Diehl, 1989: 13-14). Tradicionalmente se consideraron los causantes del fuego que afectó al centro ceremonial a fines de Metepec y los que generan un tipo de cerámica completamente distinto al complejo clásico teotihuacano (Millon, 1973, 1988).

10 Desde la fase Xolalpan tardío, la población comienza a disminuir y grupos de personas abandonan la ciudad para asentarse en otros lugares, uno de ellos pudo ser el valle de Toluca, que experimenta entonces una aceleración de su crecimiento demográfico. En la última fase del período Clásico hay un menor control de Teotihuacan sobre el valle de Toluca; en sitios como Ojo del Agua, situado al pie de la futura fortaleza de Teotenango, se elaboran cerámicas locales que imitan los estilos teotihuacanos y se distribuyen en una amplia área. Cuando la era teotihuacana llega a su fin, solamente el sitio de Santa Cruz Atzapotzaltongo desaparece; el resto de los sitios permanece habitado e incluso aumenta notablemente de extensión. En los inicios del Epiclásico la población pudo haberse cuadruplicado, creándose numerosos sitios nuevos (González de la Vara, 1999: 194).

11 Chadwick argumenta que la presencia de gentes hablando el Chochopopoloca en San Juan de Teotihuacan durante la Colonia es sintomático de situaciones anteriores.

12 Estos otomíes serán aculturados por los habitantes del centro de San Miguel Amantla en Azcapotzalco, los denominados Amanteca-teotihuacanos mencionados en algunas fuentes tardías. Estos Amanteca-teotihuacanos produjeron figurillas con tocados muy adornados con plumas, incensarios y grandes vasos adornados con figuras de dioses que tienen pervivencias de fases clásicas teotihuacanas. Otras influencias otomíes se deben percibir en la futura cerámica coyotlatelco que pudo ser hecha por los Tepanecas (también de origen otomí) (Jiménez Moreno, 1966: 52-53; 1999: 1-6).

13 Distintas evidencias de arquitectura propiamente Epiclásica en el valle de México en donde se identifican asentamientos ex novo con arquitectura cívica-ceremonial y habitacional con características propias y diferentes de las teotihuacanas. Para Patiño el Epiclásico es la persistencia de la tradición clásica, las tradiciones indígenas y la presencia de tradiciones exóticas en el centro de México, algunas de ellas muy probablemente de tradición norteña (Patiño, 1994: 200).

14 The result would have been an increase in the aggregate demand for cotton at the same time that non-local cotton produced in areas such as the Gulf Coast may have been less readily avalaible due to decreased interregional trade. The appearance of mores specialized spinning equipment within Epiclassic sites in Morelos may reflect an increase in the demand and sacle of cotton production in Central Mexico (Smith y Hirth 1988: 355).Sin embargo, quisiera matizar que en Teotihuacan durante el Clásico se identifican numerosas agujas de hueso, pero los malacates parecen ser menos funcionales.

15 El Tlalocan era la tierra de los tlaloques, dueños de las montañas y de las cuevas. Graulich menciona que los elegidos de Tlaloc eran inhumados en una cueva y que las víctimas que eran ofrecidas a este dios a menudo eran enterradas en cuevas o lanzados a las aguas (Graulich, 1990: 270).

16 No tan solo se identifican huesos de perros en los entierros, sino que Manzanilla menciona la existencia de huesos de roedores y liebres, ardilla, conejo, serpientes, diversas aves, musaraña, murciélago e incluso un maxilar de zorrillo (Manzanilla y Arrellín, 1999c: 445-446). La asociación de los restos de dichos animales con diversos individuos fechados en contextos de Coyotlatelco tardío a Mazapa, indicarían que forman parte del mismo complejo cósmico aunque con papeles diferentes. La mayoría de estos animales se relacionan con el inframundo, aunque no todos se asocian a la misma deidad. 
